IdeAs

Idées d'Amériques

$17 \mid 2021$

Villes et culture dans les Amériques

\title{
Introduction : The American Library in Paris, mémoire et observatoire des transferts culturels transatlantiques depuis 1919
}

Anne Reynès-Delobel

(2) OpenEdition

Journals

Édition électronique

URL : https://journals.openedition.org/ideas/11104

DOI : 10.4000/ideas. 11104

ISSN : 1950-5701

Éditeur

Institut des Amériques

Référence électronique

Anne Reynès-Delobel, «Introduction: The American Library in Paris, mémoire et observatoire des transferts culturels transatlantiques depuis 1919 », IdeAs [En ligne], 17 | 2021, mis en ligne le 01 mars 2021, consulté le 05 juin 2021. URL : http://journals.openedition.org/ideas/11104 ; DOl : https:// doi.org/10.4000/ideas. 11104

Ce document a été généré automatiquement le 5 juin 2021.

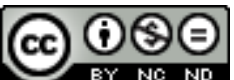

IdeAs - Idées d'Amériques est mis à disposition selon les termes de la licence Creative Commons Attribution - Pas d'Utilisation Commerciale - Pas de Modification 4.0 International. 


\title{
Introduction : The American Library in Paris, mémoire et observatoire des transferts culturels transatlantiques depuis 1919
}

\author{
Anne Reynès-Delobel
}

1 Par un heureux concours de circonstances, ce dossier, élaboré à l'occasion du centenaire de la fondation de l'American Library in Paris (ALP), partage la rubrique "Éclairages " de ce numéro d'IdeAs, Idées d'Amérique avec celui consacré aux études américaines en Europe, sous la direction de Serge Jaumain et Sonia Lehman-Frisch. De fait, la création en mai 1920 d'une bibliothèque de langue anglaise dans la capitale française répondait en partie à la volonté de pérenniser une opération culturelle initiée par des universitaires américains et menée en étroite collaboration avec les universitaires français dans le cadre de l'American University, fondée en 1919 pour accueillir les étudiants-soldats du corps expéditionnaire américain. Cette entreprise, qui participait de la visée éducative d'envergure du Library War Service, établi en 1917 par l'American Library Association (ALA) dans le but d'acheminer des livres aux soldats américains engagés dans le conflit, a considérablement renforcé et dynamisé le réseau d'échanges et de coopération universitaire entre les États-Unis et la France. Si l'ALP est aujourd'hui un pôle de ressources documentaires important pour les américanistes en France, elle est également devenue un vecteur institutionnel incontournable des transferts culturels entre les deux pays. Ce dossier, confié à quatre spécialistes américains et français, porte des regards croisés et complémentaires sur sa création en vue d'enrichir les connaissances historiques, sociologiques et bibliothéconomiques existantes et de susciter ainsi l'intérêt d'un large public.

En ouverture, Audrey Chapuis revient sur l'histoire de la bibliothèque dans l'entredeux-guerres en contrepoint de la crise sanitaire actuelle pour souligner la capacité de résilience et d'adaptation de son personnel, ainsi que les leçons tirées du passé. Nissa Ren Cannon décrit ensuite le rôle crucial joué par les journalistes du bureau parisien du 
Chicago Tribune lors du sauvetage financier ayant permis in extremis la création de l'ALP. Sa contribution permet ainsi une contextualisation plus fine de la fondation de cette institution, au croisement de l'histoire de l'imprimé et de celle des réseaux de sociabilité transatlantiques dans l'immédiat après-guerre. Cara S. Bertram revient quant à elle de manière précise et documentée sur les liens institutionnels irréfragables entre l'ALP et l'ALA. Son article s'assortit d'un précieux guide pour naviguer au sein des archives de l'association. Enfin, Rodolphe Leroy propose de faire un pas de côté pour resituer la création de l'ALA dans le cadre de l'American University et découvrir le rôle joué par deux "passeurs» essentiels, John Erskine et son épouse, Helen Worken Erskine.

3 Mémoire des transferts culturels transatlantiques, l'ALP est aussi un observatoire privilégié des relations entre deux pays qui se connaissent mal. Par-delà son histoire factuelle, l'analyse de son organisation et des représentations mentales qu'elle engage, notamment au plan de l'identité nationale, reste encore à faire. À cet égard, il est à souhaiter que ce dossier suscite des vocations.

\section{AUTEUR}

\section{ANNE REYNÈS-DELOBEL}

Anne Reynès-Delobel est maître de conférences HDR au département d'anglais d'Aix-Marseille Université et membre du LERMA ER 853. Sa recherche porte sur la littérature moderniste et les avant-gardes transatlantiques pendant l'entre-deux-guerres. anne.reynes@univ-amu.fr 